\title{
Medroxyprogesterone Acetate
}

National Cancer Institute

\section{Source}

National Cancer Institute. Medroxyprogesterone Acetate. NCI Thesaurus. Code C1155.

A synthetic, acetate derivative of the sex hormone progesterone. Medroxyprogesterone 17-acetate (NCI04) 